Rapid Reviews COVID-19

\title{
Review 2: "Going Viral: The Social Credit System and COVID-19"
}

Marianne von Blomberg 1

${ }^{1}$ University of Cologne: Universitat zu Koln, Chair for Chinese Legal Culture, Germany

Published on: Feb 22, 2021

License: Creative Commons Attribution 4.0 International License (CC-BY 4.0). 


\section{$\underline{\text { RR:C19 Evidence Scale rating by reviewer: }}$}

- Strong. The main study claims are very well-justified by the data and analytic methods used. There is little room for doubt that the study produced has very similar results and conclusions as compared with the hypothetical ideal study. The study's main claims should be considered conclusive and actionable without reservation.

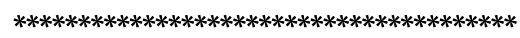

\section{Review:}

The paper at hand presents a curious inquiry into the application of the Social Credit System during a crisis, shedding much-needed light on the present utility of what has by many wrongly been perceived as a fully-fledged surveillance tool. Knight and Creemers consult the pandemic in the People's Republic of China as testing ground for the Social Credit System's mechanisms and demonstrate how the original idea of the system as a solution to non-criminal deviant behavior on the market is expanding to become a more comprehensive and flexible tool for the enforcement of whatever government priorities achieve legal status of some level. The accurate and up-to-date account of the Social Credit System they offer itself is a rare find in English language literature.

The paper asks: "how have Chinese authorities across different levels of government instrumentalized social credit as part of their response to COVID-19, and how is the crisis influencing the further development of these systems?" Knight and Creemers mainly draw on open-source government documents, a method that might strike observers who by erroneous media reports have been led to assume the Social Credit System is a highly sensitive and hence censored issue within the People's Republic as not convincing. In the authors' defense, it should be pointed out that social credit is discussed online comparatively openly, and in fact, well-documented online by the authorities - to the extent that the policy documents, laws, and news (intentionally) paint a much more comprehensive and sounder picture of it than the multiplicity of decentralized local and central, corporate and governmental efforts on the ground account for. The paper's methodological approach thus is well fit to the purpose of revealing responses to the pandemic by the Social Credit System project in its entirety.

The paper maps the diverse array of social credit mechanisms at several levels and realms as to the response they offer to pandemic control. They argue that on the one hand, the fragmentation is conducive for local leaders to swiftly react and instrumentalize their respective social credit mechanisms at hand if in need. On the other hand, the lack of unity hampers the trans-regional response that a pandemic might necessitate. Most notably, their perspective on COVID-19 as providing a 'stress test' for the Social Credit System brings to light what currently are the most troublesome 
construction sites in its creation: Data quality and quantity, its transmission as well as the technical compatibility of different systems and databases. Their valuable finding that "COVID-19 has, however, broken down some of these barriers and accelerated the process of integration" deserves further research.

On top of these valuable contributions made, the paper strives to derive insights from the study of the Social Credit System's application in fighting the pandemic about the government's overall program of 'informatization' and smart state-building. Can we learn about the state of China's 'smart state' by observing the Social Credit System's response to the pandemic? To the extent informatization endeavors cover data mobility, yes. However, as the authors recognize, the Social Credit System is not first and foremost an offspring of the government's informatization initiative. The paper oscillates between describing responses of social credit mechanisms to the pandemic (e.g. blacklisting of selling counterfeit face masks) on the one hand, and applications of technology to counter the pandemic (e.g. 'Health Code' apps) on the other; debates about how the Social Credit System draws morals into law on the one hand, and debates on privacy concerns regarding the health codes on the other. If indeed "[t]he SCS provides a microcosm [...] of the trade-offs and considerations that inevitably arise when such ideas [smart state etc.] are put into practice", tracing connections between the two and pointing out causalities between both phenomena and conflicts of interests among the actors involved would have strengthened the authors' interesting argument about China's evolving smart state. Perhaps other government initiatives or particular components of the Social Credit System which are more techheavy (but, admittedly, less sexy) might be the more informative observation objects.

Lastly, I want to further reinforce the authors' suggestion to devote future research to the public response to instances of Social Credit System instrumentalization for crisis management or other policy goals. In conjunction with it, the types of law violations that are drawn into the Social Credit System and thus endowed with its enforcement superpowers may be monitored to better understand its limitations.

To summarize, the authors have successfully harvested the occasion of the pandemic to point out recent development dynamics of the Social Credit System as well as its limits and capabilities as a policy instrument in times of crisis. That said, their situating this within the smart state building at large deserves more clarification. I endorse the timely publication of this fascinating and methodologically strong study. 